criteria, including serum level of lgG4 above $1.35 \mathrm{~g} / \mathrm{l}$ and abundant $\mathrm{lgG} 4^{+}$plasma cell infiltration in the affected tissue.

A retrospective analysis of the clinical and pathological features of 85 patients in Japan with Mikulicz's disease and related disorders identified 64 cases of IgG4+MOLPS. In comparison with 31 patients who met the diagnostic criteria for SS, those with IgG4+MOLPS had a significantly lower incidence of symptoms typical of SS, such as xerostomia, xerophthalmia and arthralgia, as well as of immunological markers, such as rheumatoid factor and antibodies against Ro/SSA and La/SSB. Furthermore, although the distribution of organ involvement in the two disorders is similar, the substantial plasmacytic infiltration in the tissue of patients with IgG4+MOLPS was characterized by an $\operatorname{lgG} 4^{+} / \operatorname{lgG}$ ratio $>50 \%$. The authors also highlighted that lgG4+MOLPS occurs with a far higher frequency in men than does SS, and, crucially, IgG4+MOLPS usually responds well to treatment with glucocorticoids.

The implications for appropriate treatment highlight the potential importance of distinguishing lgG4+MOLPS from SS, but the observations of this study will first need to be confirmed in a larger group of patients.

Original article Masaki Y et al. (2008) Proposal for a new clinical entity, IgG4-positive multi-organ lymphoproliferative syndrome: analysis of 64 cases of IgG4-related disorders. Ann Rheum Dis [doi:10.1136/ard. 2008.089169]

\section{Novel anti-CD20 monoclonal antibody shows promise as therapy for RA}

The results from the first human trial of ocrelizumab, a humanized anti-CD20 monoclonal antibody, in the treatment of rheumatoid arthritis (RA) have recently been presented. Genovese et al. investigated the safety and efficacy of ocrelizumab in 237 patients with active, moderate to severe RA who had shown an inadequate response to therapy with $\leq 6$ DMARDs. Patients were receiving concomitant methotrexate, and were randomly allocated to receive two infusions of $10,50,200,500$ or $1,000 \mathrm{mg}$ ocrelizumab or placebo.

A robust clinical response was seen across all dosages, with greater benefits in those receiving doses $\geq 200 \mathrm{mg}$. Rapid B-cell depletion was observed after the first infusion across all treatment groups, with a trend towards earlier B-cell recovery in those receiving 10 or $50 \mathrm{mg}$ ocrelizumab. Reductions in C-reactive protein levels were greatest in the high-dose groups. Doses of $200 \mathrm{mg}$ or higher had very low immunogenicity compared with the lower doses. Serious adverse events occurred in $17.9 \%$ of ocrelizumab-treated patients and $14.6 \%$ of the placebo group. Rates of serious infection were similar in both groups $(2.0 \%$ versus $4.9 \%)$. Rates of infusion were similar across all treatment groups, and were higher than the rates in the placebo group (51\% versus 17\%).

These results suggest that ocrelizumab, at doses of $200 \mathrm{mg}$ and over, combined with methotrexate could be an effective therapeutic strategy for patients with treatment-refractory RA.

Original article Genovese MC et al. (2008) Ocrelizumab, a humanized anti-CD20 monoclonal antibody, in the treatment of patients with rheumatoid arthritis. A phase I/II randomized, blinded, placebo-controlled, dose-ranging study. Arthritis Rheum 58: 2652-2661

\section{Ten-second RAPID3 tool categorizes RA disease severity similarly to standard indices}

The Routine Assessment of Patient Index Data 3 (RAPID3) self-report questionnaire aims to provide an informative, quantitative index of disease activity in patients with rheumatoid arthritis (RA) without necessitating a timeconsuming, formal joint count on the part of the physician. A recently published study by Pincus and colleagues showed that the categorization of disease severity as high, moderate, low or near-remission by RAPID3 scores was similar to that by standard indices.

In the study, 285 patients from three clinics completed the multi-dimensional health assessment questionnaire of pain, function and patient global estimate before undergoing evaluation for erythrocyte sedimentation rate, 28-joint count and physician global estimate. RAPID3 correlated significantly with both CDAI (Clinical Disease Activity Index; $\rho=0.74, P<0.001$ ) and DAS28 (Disease Activity Score 28; $\rho=0.66$, $P<0.001)$. Adding additional measures to the calculation of RAPID3 scores, such as joint count or physician global assessment, increased this correlation, but not enough to justify the additional examination time required. 